\section{Caracterización de cepas de Campylobacter jejuni obtenidas desde carne de pollo y heces de aves de corral de la zona central de Chile}

\author{
SINDY GUTIÉRREZ ${ }^{1, \mathrm{a}}$, DANIEL ORELLANA ${ }^{1, \mathrm{~b}}$, \\ CLAUDIO MARTÍNEZ ${ }^{1,2, \mathrm{c}}$, VERÓNICA GARCÍA MENA ${ }^{1,2, \mathrm{~d}}$
}

\section{Characterization of Campylobacter jejuni samples coming form poultry meat and feces}

Background: Campylobacter jejuni is one of the main causal agents of food borne diseases. Infections with this pathogen are mainly caused by chicken meat consumption. Aim: To characterize antibiotic resistance and virulence factors in C. jejuni strains obtained from chicken meat and poultry feces in Central Chile. Material and Methods: The presence of C. jejuni in 30 meat and 40 feces samples from poultry was studied. From these samples, we obtained 40 strains which were characterized at the molecular level for the presence of 16 genes involved in virulence using PCR. In parallel, antibiotic resistance for ciprofloxacin, nalidixic acid, tetracycline, erythromycin, azithromycin, chloramphenicol y ampicillin was analyzed. Results: Twenty and 63\% of feces and chicken meat samples were positive for C. jejuni, respectively. Moreover, a high percentage of strains showed antibiotic resistance, where $27 \%$ of strains were resistant to all tested antibiotics, except for azithromycin. Finally, 10\% of the strains coming from feces contained 14 out of 16 virulence genes evaluated. Only $23 \%$ of the strains did not contain any of these genes. Conclusions: A high percentage of feces and chicken meat samples are contaminated with $C$. jejuni. Moreover, these strains show a high genetic and phenotypic diversity represented by their antibiotic resistance profiles and the presence of virulence factors.

(Rev Med Chile 2017; 145: 1551-1558)

Key words: Anti-Bacterial Agents; Campylobacter jejuni; Poultry; Poultry Products.

\author{
'Departamento de Ciencia y \\ Tecnología de los Alimentos, \\ Facultad Tecnológica, Universidad \\ de Santiago (USACH). Santiago, \\ Chile. \\ ${ }^{2}$ Centro de Ciencia y Tecnología \\ de los Alimentos Universidad de \\ Santiago. Santiago, Chile. \\ a Médico veterinario. \\ bIngeniero de Alimentos. \\ 'Doctor en Ciencias, mención \\ Biología. \\ ${ }^{\mathrm{d} B i o q u i ́ m i c a . ~ P h D . ~}$ \\ Fuente de financiamiento: \\ Proyecto financiado por \\ Fondecyt 11130148 y Proyecto \\ de inserción a la academia \\ 791220024. \\ Autores declaran no tener \\ conflictos de interés. \\ Recibido el 6 de enero de 2017, \\ aceptado el 28 de diciembre de \\ 2017. \\ Correspondencia a: \\ Verónica García Mena \\ Departamento de Ciencias y \\ Tecnología de los Alimentos. \\ Facultad Tecnológica. Universidad \\ de Santiago de Chile. \\ Obispo Manuel Umaña 050. \\ Edificio de Alimentos. Estación \\ Central, Santiago, Chile. \\ verónica.garcia@usach.cl
}

ampylobacter jejuni es una de las principales bacterias que causa gastroenteritis en países industrializados ${ }^{1}$. En Chile, Campylobacter es el tercer agente causal de diarreas en niños ${ }^{2,3}$. Es adquirida por consumo de leche no pasteurizada, aguas contaminadas, etc. Sin embargo, se encuentra en altas densidades en el intestino de las aves de corral, como consecuencia, el consumo de carne de ave mal cocida es responsable de $50-80 \%$ de los casos de campylobacteriosis ${ }^{4}$.

La campylobacteriosis es una gastroenteritis aguda, autolimitada ${ }^{5} \mathrm{y}$ habitualmente no requiere tratamiento. Sin embargo, en pacientes inmunocomprometidos o con infecciones prolongada es necesario el tratamiento con antibióticos ${ }^{6}$, siendo las fluoroquinolonas y los macrólidos los antibióticos comúnmente seleccionados, a pesar del aumento de cepas resistentes a estos ${ }^{1,7}$.

En este trabajo caracterizamos la susceptibilidad de 40 cepas de $C$. jejuni, obtenidas de heces de aves de corral de crianza doméstica (14) y de carne de pollo de consumo humano (26), a antibióticos de distintas familias, entre ellos macrólidos (eritromicina y azitromicina), por el aumento de 
cepas resistentes, y quinolonas (ácido nalidíxico y ciprofloxacina) por ser utilizado en la industria avícola. Además, evaluamos la presencia de genes involucrados en virulencia, incluyendo genes que participan en adhesión, invasión, sobrevivencia, producción de toxina y en transporte de sideróforo. Estos genes entregan una panorámica general de la virulencia ${ }^{8,9}$, aunque los genes que participan en la infección de Campylobacter involucran otros procesos.

\section{Materiales y Métodos}

\section{Cepas bacterianas y medios de cultivos}

Las 40 cepas analizadas fueron obtenidas a partir de heces de aves de corral o carne de ave disponible a la venta según los protocolos descritos anteriormente ${ }^{10}$. Las muestras de carne de pollo fueron obtenidas de supermercados (15 muestras) y carnicerías (15 muestras) de las comunas de Estación Central, Quinta Norma y Santiago Centro, mientras que 40 muestras de heces fueron obtenidas de aves de crianza doméstica de las comunas de Talagante, Casablanca, Melipilla y Cauquenes. Diluciones de las muestras fueron sembradas en agar selectivo mCCDA (Oxoid) y posteriormente incubadas a $42^{\circ} \mathrm{C}$ por $48 \mathrm{~h}$ en condiciones de microaerofilia. Posteriormente, se tomaron a lo menos 8 colonias de cada muestra positiva y fueron cultivadas en agar tripticasa de soya $5 \% \mathrm{v} / \mathrm{v}$ sangre de cordero. Posteriormente, se analizó la morfología de las bacterias por microscopía ${ }^{10}$ y aquellas seleccionadas se cultivaron en agar tripticasa de soya $5 \% \mathrm{v} / \mathrm{v}$ sangre de cordero para posteriores análisis. Las colonias obtenidas en esta etapa son denominadas aislados y se almacenaron a $-80^{\circ} \mathrm{C}$ en caldo Brucella 30\% v/v de glicerol.

\section{Identificación de cepas de C. jejuni}

Las cepas de C. jejuni fueron identificadas a través de la prueba de hidrólisis del hipurato y confirmadas por reacción en cadena de la polimerasa $(\mathrm{PCR})^{11}$. Con el fin de establecer que las colonias correspondían a cepas diferentes se realizó un RAPD (del inglés Random Amplification of Polymorphic DNA) usando el primer AP4 $4^{12}$. Los perfiles electroforéticos fueron analizados utilizando el programa GelJ v 2,0. ${ }^{13}$ La identificación de cepas se realizó por el coeficiente de correlación de Dice y el algoritmo de clustering UPGMA (del inglés
Unweighted Pair-Group Method with Averages) con una tolerancia de optimización de 5\%. Aquellos aislados que tenían un perfil de RAPD particular y distinto a otros fueron denominados cepa.

\section{Ensayo de susceptibilidad antimicrobiana}

Se analizó la susceptibilidad a los antibióticos mediante dilución en agar para los antibióticos cloranfenicol y eritromicina, epislometría (E-test) para azitromicina, ampicilina y eritromicina y sensidiscos para ciprofloxacina, tetraciclina, eritromicina y ácido nalidíxico. Los puntos de corte para definir una cepa susceptible, resistente o intermedia se definieron según lo descrito anteriormente ${ }^{14,15}$. Se utilizó el método sensidiscos y E-test por su fácil implementación, además, E-test es cuantitativo. En el caso de eritromicina se utilizó, también, dilución en agar para confirmar los resultados obtenidos con otros métodos.

\section{Análisis de PCR de genes de virulencia}

Un total de 16 parejas de partidores fueron utilizados para evaluar la presencia de genes de virulencia: adhesión y colonización (flaA, flaC, cadF, dnaJ, cbrR), invasión y virulencia (virB11, ciaB, pldA), sobrevivencia ( $r a c R, \operatorname{sod} B, h \operatorname{tr} A, \operatorname{clp} A)$, producción de toxina $(c d t A, c d t C)$ y transportadores de sideróforos $(\mathrm{ceuE})$ y wlaN por mimetizar a los gangliosidos ${ }^{16}$. En este estudio no fue incluido el gen $c d t B$, debido a que no fue posible estandarizar su amplificación. Como control positivo se utilizó ADN (ácido desoxirribonucleico) de las cepas de referencias de C. jejuni ATCC33560, NCTC13367 y ATCC43430. Como control negativo se utilizó ADN de Escherichia coli ATCC25922.

\section{Resultados}

\section{Identificación de cepas de C. jejuni}

Los resultados indican que $63,3 \%$ de las muestras de carne resultaron positivas para Campylobacter, las cuales provienen de carnicería (8) y supermercados (11). Además, $20 \%$ de las muestras de heces fueron positivas (Tabla 1).

A partir de cada muestras positivas para Campylobacter se seleccionaron entre 8 y 20 colonias y se analizaron por PCR con el fin de determinar la especie ${ }^{11}$. En esta etapa cada una de las colonias fue tratada en forma independiente y corresponden a un aislado. Posteriormente, y con el fin de 
determinar perfiles genéticos distintos e identificar cepas se realizó RAPD (Figura 1). Así, colonias obtenidas de una misma muestra con perfiles de RAPD diferentes corresponde a cepas distintas. De esta forma se obtuvo 151 aislados, de los cuales 75 provienen de heces y 76 de carne de pollo. Luego del análisis de RAPD, se determinó que 145 eran cepas distintas, pues poseían un perfil genético distinto. En la muestra 7 (heces provenientes de Talagante) se obtuvo un total de 17 aislados, de las cuales, 13 de ellas se encontraban muy cercanas en el árbol filogenético (dato no mostrado). Por otro lado, de los 5 aislados obtenidos en la muestra 47 (carne proveniente de supermercado) se observaron 3 cepas distintas molecularmente, aunque muy cercanas. Esta observación indica que una muestra de carne o de heces puede estar contaminada por más de una cepa de $C$. jejuni.

A partir de estos resultados se seleccionaron 40 cepas: 14 cepas obtenidas desde heces y 26 desde carne de pollo.

Resistencia antibióticos de cepas de C. jejuni.

A las cepas antes seleccionadas, se evaluó la resistencia a los antibióticos eritromicina, ciprofloxacina, tetraciclina, ácido nalidíxico, azitromicina, ampicilina y cloranfenicol.

Los resultados indican que $27 \%$ de las cepas, todas obtenidas de carne, eran resistentes a todos los antibióticos analizados, excluyendo azitromicina (Figura 1 y Tabla 2). Por otro lado, ninguna de las cepas obtenidas desde heces fue resistente a cloranfenicol (MIC $\geq 32$ ), y mostraban MIC (concentración inhibitoria mínima) que variaban
Tabla 1. Muestras contaminadas con C. jejuni

\begin{tabular}{|lccc|}
\hline & $\begin{array}{c}\text { n de } \\
\text { muestras }\end{array}$ & Positivas & \% Positivas \\
\hline Heces & 40 & 8 & 20 \\
\hline Carne de pollo & 30 & 19 & 63,3 \\
Total & 70 & 27 & 38,6 \\
\hline
\end{tabular}

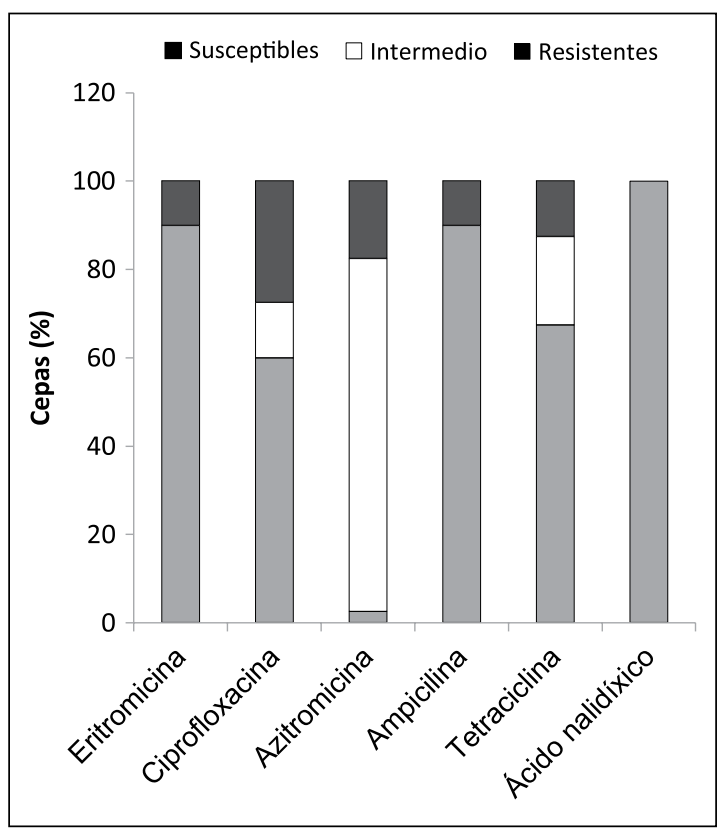

Figura 1. Distribución de las cepas de C. jejuni respecto a su susceptibilidad a los antibióticos. Clasificación de las 40 cepas analizadas para los antibióticos. En negro: cepas susceptibles, en blanco: cepas con susceptibilidad intermedia, en gris: cepas resistentes.

Tabla 2. Resultados de susceptibilidad a los antibióticos de cepas de C. jejuni analizados por dilución en agar, E- test o sensidiscos

\begin{tabular}{|lccccc|}
\hline Antibiótico & \% R (MIC) & \% R (E-test) & \multicolumn{2}{c}{ Distribución de los resultados se sensidiscos (\%) } \\
\hline Eritromicina & 87,5 & 90,0 & 2,5 & 10 & R \\
\hline Tetraciclina & 77,1 & ND & 17,5 & 12,5 & 70 \\
\hline Cloramfenicol & 55,3 & ND & ND & ND & ND \\
\hline Ciprofloxacina & ND & ND & 27,5 & 12,5 & 60 \\
\hline Ácido nalidíxico & ND & ND & 30,0 & 0 & 70 \\
\hline Ampicilina & ND & 90,0 & ND & ND & ND \\
\hline Azitromicina & ND & 2,5 & ND & ND & ND \\
\hline
\end{tabular}

ND: No determinado, S: Susceptible, I: Intermedio, R: Resistente. 


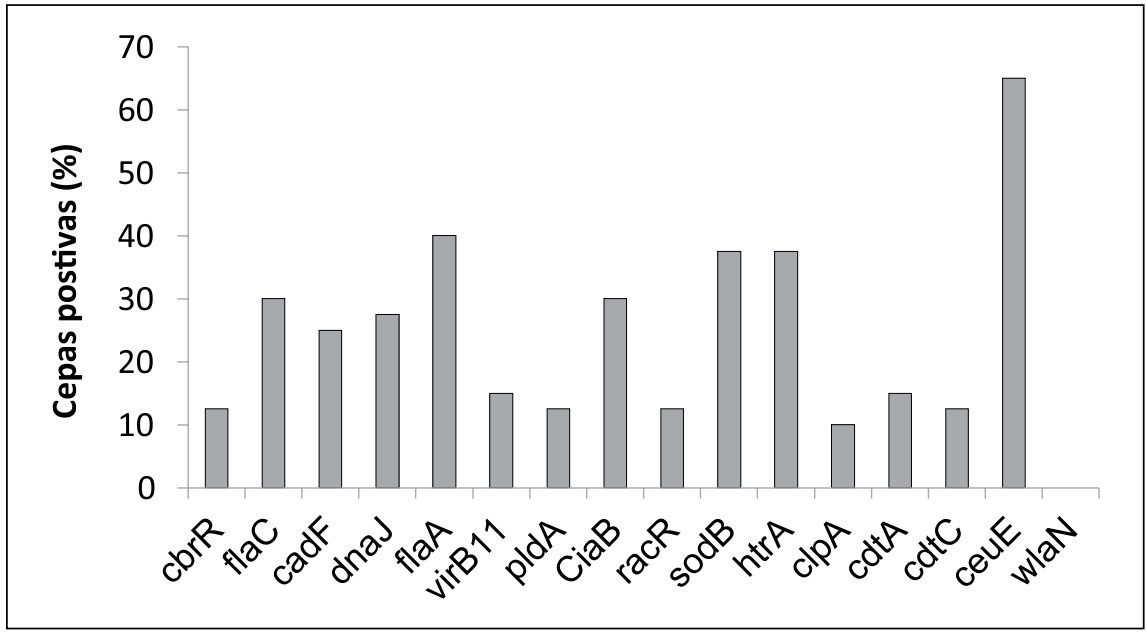

Figura 2. Presencia de genes relacionados con virulencias de $C$. jejuni. En las 40 cepa seleccionadas y mediante PCR se analizó la presencia de 16 genes importantes para la virulencia de $C$. jejuni. entre 4 y $8 \mu \mathrm{g} / \mathrm{mL}$, por lo cual se clasificaron en el rango intermedio (entre 0,12 y $32 \mu \mathrm{g} / \mathrm{mL}$ ). Por otro lado, $77 \%$ de las cepas obtenidas desde carne fueron resistentes a este antibiótico.

Los resultados de MIC mediante el método de dilución en agar para eritromicina mostraron un alto porcentaje de cepas resistentes (87,5\%). Para confirmar esta observación se realizó el análisis de susceptibilidad de las cepas mediante sensidiscos y E-test, observando que entre 87,5 y $90 \%$ de las cepas eran resistentes a eritromicina. Las cepas $\mathrm{Cj} 123$ y Cj48 resultaron con MIC de $\leq 0,125$ y $0,5 \mu \mathrm{g} / \mathrm{mL}$ respectivamente, mediante el método de dilución en agar. Sin embargo, en el ensayo de E-test su MIC fue de $\geq 256$ y $64 \mu \mathrm{g} / \mathrm{mL}$, respectivamente, y por sensidiscos no presentaban halo de inhibición, por lo cual fueron clasificadas como resistentes.

Por otro lado, $60 \%$ de las cepas fueron resistentes a ciprofloxacina, todas provenientes de carne de pollo. Las cepas obtenidas desde heces presentaban halos de inhibición que variaban entre 21 y $40 \mathrm{~mm}$, por lo tanto se clasificaron como susceptible o intermedio. Respecto a ácido nalidíxico, solo una cepa proveniente de carne de pollo resultó susceptible. Sin embargo, en cepas provenientes de heces se observó más variedad, observando 3 cepas resistentes: la cepa Cj32 no presentaba halo de inhibición y las cepas Cj61 y Cj36 presentaban un halo de $14 \mathrm{~mm}$. Por otro lado, las cepas provenientes de heces resistentes a ácido nalidíxico fueron de susceptibilidad intermedia a ciprofloxacina.
En cuanto a azitromicina, del total de muestras 2 cepas fueron resistentes, con un MIC de 64 y $32 \mu \mathrm{g} / \mathrm{mL}$. Finalmente, para ampicilina, todas las cepas de carne fueron resistentes y en cepa de heces, 4 cepas resultaron susceptibles.

\section{Factores de virulencia}

Se evaluó la presencia de genes relacionados con virulencia en las cepas obtenidas observando que el gen más frecuente fue ceuE (en 62,5\% de las cepas). Por otro lado, ninguna cepa presentó el gen wlnA. Las cepas Cj22, Cj28, Cj29 y Cj32, provenientes de heces, fueron las que presentaron mayor número de genes (14 de 16). Además, estas cepas fueron las más susceptibles a los antibióticos, excepto la cepa Cj22 que fue resistente a azitromicina. Por otro lado, la cepa Cj119 fue la cepa proveniente de carne que mostró mayor presencia de genes de virulencia ( 10 de 16). Además, 9 cepas no presentaron ninguno de los genes evaluados, 5 cepas de heces y 4 de carne (Figura 2).

\section{Discusión}

C. jejuni es una bacteria que se encuentra en alta concentración en el tracto digestivo de las aves de corral y, en consecuencia, una alta cantidad de carcasas de pollos se contaminan ${ }^{17,18}$.

En este estudio se observó que nuestro país no es la excepción. Así, 63,3\% de las muestras de carne de pollo resultaron positivas para esta bacteria. Estudios anteriores indican que durante 
la evisceración se produce la mayor contaminación, alcanzando $54 \%$ de carcasas contaminadas, aunque el proceso de congelamiento disminuye la cantidad de bacterias, el número de carcasas contaminadas se mantiene ${ }^{19}$. Este porcentaje de carne contaminada es similar a lo reportado en otros países: en Canadá, $62 \%$ de las muestras de carne de pollo resultaron positivas para Campylobacter $^{20}, 49,5 \%$ en España ${ }^{21}$ y en $83,3 \%$ en el Reino Unido ${ }^{22}$. En América del Sur, la situación es similar: en Argentina ${ }^{23} 87,8 \%$ de las carnes de pollo presenta C. jejuni, 68,8\% en Brasil ${ }^{24}$ y en Chile, entre 54 y $90 \%{ }^{19}$. Asimismo, $92,9 \%$ de hígados de pollo reportan la presencia de Campylobacter ${ }^{25}$.

Por otro lado, 20\% de las muestras de heces fueron positivas para $C$. jejuni, estos resultados son similares a los reportados en el sur de Chile, en donde 34\% de las heces de aves presentan $C$. jejuni $i^{26}$ y $25,7 \%$ de heces de gallinas presentan Campylobacter $s p^{27}$ y entre $45 \%$ y $100 \%$ en otras aves $^{28}$. Respecto al tipo de crianza de las aves, se ha observado una mayor prevalencia de Campylobacter sp. en crianza doméstica, respecto a aves de poblaciones confinadas ${ }^{29}$.

Por otro lado, se ha descrito que las carcasas de pollo pueden estar contaminadas con más de una cepa de C. jejuni $i^{30}$, lo cual fue confirmado con nuestros resultados, pues muestras de heces y de carne tienen más de una cepa, según el patrón molecular observado por RAPD. Así, 50\% de las muestras positivas de heces tiene 2 cepas distintas y $89,5 \%$ de las muestras positivas de carne tienen 2 o 3 cepas distintas. Esto último puede ser reflejo de una contaminación cruzada en las vitrinas de venta de pollo a granel. Esta coexistencia de cepas ha sido observada en biopelículas ${ }^{31}$ y en pacientes con Guillian Barré ${ }^{32}$.

Por otro lado, los resultados muestran un porcentaje alto de cepas resistentes a ciprofloxacina en cepas obtenidas desde carne de pollo, similar a lo observado anteriormente ${ }^{35,36}$. Esto se relaciona con un aumento de las cepas resistentes a este antibiótico en pacientes. En Chile se ha descrito un aumento en las cepas resistentes a este antibiótico obtenidas coprocultivos de pacientes en Santiago $\left(32,4 \%\right.$ durante el año 2008) ${ }^{37}$, lo que difiere de lo observado años atrás en el sur de Chile, en donde el 100\% de los aislados clínicos eran susceptibles a este antibiótico ${ }^{38}$. De manera similar en Beijing, China, se describió la resistencia a antibióticos de aislados clínicos desde el año 1994 al 2010, observando un aumento en la resistencia a ciprofloxacina en el tiempo, esto mismo se observó con tetraciclinas $\mathrm{y}$, en forma menos importante, con macrólidos $^{39}$. Nuestros resultados muestran una alta cantidad de cepas resistentes a eritromicina, preferentemente en cepas obtenidas de carne de pollo $(92,6 \%)$, lo que difiere a lo reportado anteriormente en nuestro país ${ }^{26,37}$, aunque, en los últimos años se ha reportado un aumento en las cepas resistentes a eritromicina ${ }^{39}$. Una posible explicación de esto es el pequeño número de muestras analizadas y la posibilidad de que las muestras de carne provengan de un mismo faenador. A pesar de ser adquiridos en distintos locales, todas las muestras provienen de comunas cercanas. Además, se ha descrito que la resistencia a macrólidos está determinada por varios polimorfismos ubicados en distintos genes ( $r A R N$ 23S, L4, L22, bomba CmeABC), en este sentido, son varios los blancos que pueden producir una cepa resistente a este antibiótico. Incluso se ha descrito nuevos genes, como el gen erm que codifica para rARN metilasa ${ }^{40}$ que podría participar en la resistencia a macrólidos ${ }^{39}$. Finalmente, es importante destacar que en países como Perú ${ }^{41}$ e India ${ }^{42}$ se ha descrito un aumento en las cepas de C. jejuni resistentes a eritromicina en los últimos años. Por otro lado, solo 2 cepas resultaron resistentes a azitromicina (5\% de las cepas). De igual forma, se han reportado diferentes perfiles de resistencia de cepas obtenidas en aislados clínicos y carne de pollo en distintas partes del mundo ${ }^{12,15,43,44,41}$. Estas diferencias podrían ser producto por las diferentes estrategias de control biológico usadas en la producción primaria y en salud pública.

Mediante técnicas de secuenciación masiva, se ha observado que existe una alta variabilidad entre las cepas en cuanto a la presencia de genes relacionados con virulencia ${ }^{12,16}$. Así, se ha observado que algunas cepas carecen de genes involucrados en la biosíntesis de flagelo y lipopolisacáricos, captura de hierro, modificación del ADN, etc. Esto muestra una alta plasticidad genética ${ }^{33,34}$, lo que facilita la adaptación de esta bacteria a nichos distintos, convirtiéndolo en un patógeno difícil de controlar.

Dentro de los factores de virulencia, el más frecuentemente fue $c e u E$, que codifica para una lipoproteína involucrada en el transporte de enteroquelina ${ }^{8,45}$, esencial para la infección. Algunos estudios coinciden con la alta frecuencia de este gen en distintas cepas ${ }^{46}$, sin embargo, en otros 
estudios este gen es menos abundante ${ }^{16}$. Por otro lado, virB 11 se encuentra en 15\% de las muestras, todas obtenidas desde carne de pollo, esto es ligeramente superior a lo reportado anteriormente en nuestro país $(7,3 \% \text { de las cepas })^{9}$. Otro gen que ha sido analizado en muestras chilenas, en otros trabajos, es $c d t B$, el cual estaba presente en $100 \%$ de las muestras. Sin embargo, en este artículo no fue incluido. Finalmente, el gen wlaN no fue detectado en ninguna cepa, en forma similar a lo reportado anteriormente en nuestro país ${ }^{47}$.

En resumen, la alta frecuencia de contaminación de carne de pollo con C. jejuni con cepas resistentes a uno o más antibióticos, sumado a la diversidad genética de las cepas en cuanto a la presencia de factores de virulencias confirman la importancia de vigilar, investigar y controlar $C$. jejuni en alimentos debido a su alto impacto en salud pública.

Agradecimientos: Proyecto financiado por Fondecyt 11130148 y Proyecto de inserción 791220024.

\section{Referencias}

1. Meunier M, Guyard-Nicodeme M, Dory D, Chemaly M. Control strategies against Campylobacter at the poultry production level: Biosecurity measures, feed additives and vaccination. J Appl Microbiol 2016; 120 (5): 113973.

2. Prado V. Epidemiología de la enteritis por Campylobacter en niños. Rev Med Chile 1984; 112: 1153.

3. Prado V, Martínez J, Reyes L, Ducheylard M, Bercovich M, Millán V, et al. Características de la infección intestinal por Campylobacter jejum en lactantes chilenos. Rev Med Chile 1985; 113: 521-5.

4. Miller W, Mandrell R. Campylobacter in the food and water supply: Prevalence, outbreaks, isolation, and detection. In: Ketley J, Konkel M, editors. Campylobacter jejuni: New perspectives in molecular and cellular biology. 1st ed. Norfolk: Horizon Scientific Press; 2004. p. 10963.

5. Blaser M. Epidemiologic and clinical features of Campylobacter jejuni infections. J Infect Dis 1997; 176: 103-5.

6. Engberg J, Aarestrup FM, Taylor DE, Gerner-Smidt P, Nachamkin I. Quinolone and macrolide resistance in Campylobacter jejuni and C. coli: Resistance mechanisms and trends in human isolates. Emerg Infect Dis 2001; 7 (1): 24-34.

7. Luangtongkum T, Morishita TY, El-Tayeb AB, Ison AJ,
Zhang Q. Comparison of antimicrobial susceptibility testing of Campylobacter spp. by the agar dilution and the agar disk diffusion methods. J Clin Microbiol 2007; 45 (2): 590-4.

8. González I, Grant KA, Richardson PT, Park SF, Collins MD. Specific identification of the enteropathogens Campylobacter jejuni and Campylobacter coli by using a PCR test based on the ceuE gene encoding a putative virulence determinant. J Clin Microbiol 1997; 35 (3): 759-63.

9. González-Hein G, Huaracan B, García P, Figueroa G. Prevalence of virulence genes in strains of Campylobacter jejuni isolated from human, bovine and broiler. Brazilian J Microbiol 2013; 44 (4): 1223-9.

10. Hunt JM, Abeyta C, Tran T. Charper 7: Campylobacter. In: Bacteriological Analytical Manual. 8th ed. 2012.

11. Wang G, Clark CG, Taylor TM, Pucknell C, Barton C, Price L, et al. Colony Multiplex PCR Assay for Identification and Differentiation of Campylobacter jejuni, C. coli, C. lari, C. upsaliensis, and C. fetus subsp. fetus. Society 2002; 40 (12): 4744-7.

12. Di Giannatale E, Di Serafino G, Zilli K, Alessiani A, Sacchini L, Garofolo G, et al. Characterization of antimicrobial resistance patterns and detection of virulence genes in Campylobacter isolates in Italy. Sensors (Basel) 2014; 14 (2): 3308-22.

13. Heras J, Domínguez C, Mata E, Pascual V. GelJ - a tool for analyzing DNA fingerprint gel images. BMC Bioinformatics 2015; 16: 210.

14. Clinical and Laboratory Standards Institute (CLSI). In: Methods for Antimicrobial Dilution and Disk Susceptibility Testing of Infrequently Isolated or Fastidious Bacterial; Approved Guidline-Second Edition CLSI, Wayne, PA, Document M45-A2. 2010.

15. Miflin JK, Templeton JM, Blackall PJ. Antibiotic resistance in Campylobacter jejuni and Campylobacter coli isolated from poultry in the South-East Queensland region. J Antimicrob Chemother 2007; 59 (4): 775-8.

16. Hanning I, Biswas D, Herrera P, Roesler M, Ricke S. Prevalence and characterization of Camapylobacter jejuni isolated from pasture flock poultry. J Food Sci 2010; 75 (7): 496-502.

17. FAO/WHO [Food and Agriculture Organization of the United Nations/World HealthOrganization]. In: Risk assessment of Campylobacter spp in broiler chickens: Interpretative Summary Microbiological Risk Assessment Series No 11 Geneva 35pp. 2009.

18. Neimann J, Engberg J, Molbak K, Wegener H. A case-control study of risk factors for sporadic campylobacter infections in Denmark. Epidemiol Infect 2003; 130: 353-66. 
19. Figueroa G, Troncoso M, López C, Rivas P, Toro M. Occurrence and enumeration of Campylobacter spp. during the processing of Chilean broilers. BMC Microbiol. 2009; 9 (1): 94.

20. Bohaychuk VM, Gensler GE, King RK, Manninen KI, Sorensen $\mathrm{O}, \mathrm{Wu}$ JT, et al. Occurrence of pathogens in raw and ready-to-eat meat and poultry products collected from the retail marketplace in Edmonton, Alberta, Canada. J Food Prot 2006; 69 (9): 2176-82.

21. Dominguez C, Gomez I, Zumalacarregui J. Prevalence of Salmonella and Campylobacter in retail chicken meat in Spain. Int J Food Microbiol 2002; 72 (1-2): 165-8.

22. Kramer JM, Frost J a, Bolton FJ, Wareing DR. Campylobacter contamination of raw meat and poultry at retail sale: identification of multiple types and comparison with isolates from human infection. J Food Prot 2000; 63 (12): 1654-9.

23. Giacoboni G, Tellechea D, Agostini A. Pollos Camperos Campylobacter Jejuni in a Free-Range. Analecta Vet 2002; 22 (2): 42-7.

24. Kuana S, Santos L, Rodrigues L, Borsoi A, Moraes H, Salle C, et al. Occurrence and Characterization of Campylobacter in the Brazilian Production and Processing of Broilers. Avian Dis 2008; 52 (4): 680-4.

25. Fernandez H, Pison V. Isolation of thermotolerant species of Campylobacter from commercial chicken livers. Int J Food Microbiol 1996; 29 (1): 75-80.

26. Rivera N, Bustos R, Montenegro S, Sandoval M, Castillo J, Fernández H, et al. Microbiología. Rev Chil Infectol 2011; 28 (6): 555-62.

27. Fernández H, Torres N. Occurrence of Campylobacter jejuni and Campylobacter coli in three groups of hens of different geographic origin in Southern Chile. Arch Med Vet 200AD; 32 (2): 241-4.

28. Fernandez H, Vera F, Villanueva MP. Arcobacter and Campylobacter species in birds and mammals from Southern Chile. Arch Med Vet 2007; 39 (2): 163-5.

29. Tresierra-Ayala A, Fernández H, Bendayán M, Pereyra G, Bernuy A. Aislamiento de especies termotolerantes de Campylobacter en dos poblaciones de pollos criados con y sin confinamiento. Rev Saude Publica 1995; 29 (5): 389-92.

30. Jacobs-Reitsma1 WF, Van De Giessen2 AW, Bolder 'and NM, Mulder1 RWAW. Epidemiology of Campylobacter spp. at two Dutch broiler farms. Epidemiol Infect 1995; 114: 413-21.

31. Ica T, Caner V, Istanbullu O, Nguyen HD, Ahmed B, Call DR, et al. Characterization of mono- and mixed-culture Campylobacter jejuni biofilms. Appl Environ Microbiol 2012; 78 (4): 1033-8.

32. Godschalk P, Gilbert M, Jacobs B, Kramers T, Tio-Gi- llen A, Ang C, et al. Co-infection with two different Campylobacter jejuni strains in a patient with the Guillain-Barré syndrome. Microbes Infect 2006; 8 (1): 248-53.

33. Dorrell N, Mangan J a, Laing KG, Hinds J, Linton D, Al-ghusein $\mathrm{H}$, et al. Whole Genome Comparison of Campylobacter jejuni Human Isolates Using a Low-Cost Microarray Reveals Extensive Genetic Diversity. Genome Res 2001;1706-15.

34. Leonard Ii EE, Takata T, Blaser MJ, Falkow S, Tompkins LS, Gaynor EC. Use of an Open-Reading Frame-Specific Campylobacter jejuni DNA Microarray as a New Genotyping Tool for Studying Epidemiologically Related Isolates. J Infect Dis 2003; 187: 691-4.

35. Zhang T, Luo Q, Chen Y, Li T, Wen G, Zhang R, et al. Molecular epidemiology, virulence determinants and antimicrobial resistance of Campylobacter spreading in retail chicken meat in Central China. Gut Pathog 2016; 8 (48): 1-9.

36. Ngoc T, Nguyen M, Hotzel H, Njeru J, Mwituria J, El-adawy $\mathrm{H}$, et al. Antimicrobial resistance of Campylobacter isolates from small scale and backyard chicken in Kenya. Gut Pathog 2016; 8 (1-9).

37. García P, Valenzuela N, Rodríguez V, León E, Fernández H. Susceptibilidad antimicrobiana de Campylobacter jejuni aislado de coprocultivos en Santiago de Chile. Rev Chil Infect 2009; 26 (6): 511-4.

38. Fernández H, Mansilla M, González V. Antimicrobial susceptibility of Campylobacter jejuni subsp. jejuni assessed by E-test and double dilution agar method in Southern Chile. Mem Inst Oswaldo Cruz 2000; 95: 247-9.

39. Zhou J, Zhang M, Yang W, Fang Y, Wang G, Hou F. A seventeen-year observation of the antimicrobial susceptibility of clinical Campylobacter jejuni and the molecular mechanisms of erythromycin-resistant isolates in Beijing, China. Int J Infect Dis 2016; 42: 28-33.

40. Deng F, Shen J, Zhang M, Wu C, Zhang Q, Wang Y. Constitutive and inducible expression of the rRNA Methylase gene erm(B) in Campylobacter. Antimicrob Agents Chemother 2015; 59 (10): 6661-4.

41. Pollett S, Rocha C, Zerpa R, Patiño L, Valencia A, Camiña M, et al. Campylobacter antimicrobial resistance in Peru: a ten-year observational study. BMC Infect Dis 2012; $12: 1$.

42. Mukherjee P, Ramamurthy T, Mitra U, Mukhopadhyay AK. Emergence of high-level azithromycin resistance in Campylobacter jejuni isolates from pediatric diarrhea patients in Kolkata, India. Antimicrob Agents Chemother 2014; 58 (7): 4248.

43. Wimalarathna HML, Richardson JF, Lawson AJ, Elson R, Meldrum R, Little CL, et al. Widespread acquisition 
of antimicrobial resistance among Campylobacter isolates from UK retail poultry and evidence for clonal expansion of resistant lineages. BMC Microbiol 2013; 13 (1): 160.

44. Kashoma IP, Kassem II, Kumar A, Kessy BM, Gebreyes W, Kazwala RR, et al. Antimicrobial resistance and genotypic diversity of campylobacter isolated from pigs, dairy, and beef cattle in Tanzania. Front Microbiol 2015; 6 (1): 1240.

45. Park SF, Richardson PT. Molecular characterization of a Campylobacter jejuni lipoprotein with homology to periplasmic siderophore-binding proteins. J Bacteriol 1995; 177 (9): 2259-64.
46. Bang DD, Nielsen EM, Scheutz F, Pedersen K, Handberg $\mathrm{K}$, Madsen M. PCR detection of seven virulence and toxin genes of Campylobacter jejuni and Campylobacter coli isolates from Danish pigs and cattle and cytolethal distending toxin production of the isolates. J Appl Microbiol 2003; 94 (6): 1003-14.

47. Lapierre L, Gatica M, Riquelme V, Vergara C, Yañez J, San Martín B, et al. Characterization of Antimicrobial Susceptibility and Its Association with Virulence Genes Related to Adherence, Invasion, and Cytotoxicity in Campylobacter jejuni and Campylobacter coli Isolates from Animals, Meat, and Humans. Microb Drug Resist 2016; 22 (5): 432-44. 